PROCEEDINGS OF THE

AMERICAN MATHEMATICAL SOCIETY

Volume 140, Number 2, February 2012, Pages 715-730

S 0002-9939(2011)11344-3

Article electronically published on October 5, 2011

\title{
QUASI SURE LOCAL CONVERGENCE RATE OF A BROWNIAN MOTION IN THE HÖLDER NORM
}

\author{
YONGHONG LIU AND YUHUI LI \\ (Communicated by Sergei K. Suslov)
}

\begin{abstract}
We estimate the local convergence rate of Strassen type for a Brownian motion in the Hölder norm with respect to $C_{r, p}$-capacity on an abstract Wiener space. The local convergence rate for increments of a Brownian motion in the Hölder norm with respect to $C_{r, p}$-capacity is also derived.
\end{abstract}

\section{INTRODUCTION}

The quasi sure (q.s.) analysis is one of the important concepts in stochastic analysis. It studies the properties of stochastic processes which hold outside sets with capacity null. Capacity is a kind of set function which is much finer than probability.

Let $L^{p}=L^{p}(B, \mu)$ denote the real-valued function space on $(B, \mu)$ and let $(B, H, \mu)$ be an abstract Wiener space with the Ornstein-Uhlenbeck operator $\mathcal{L}$. We denote the Sobolev space $D^{r, p}$ by $D^{r, p}=(1-\mathcal{L})^{-\frac{r}{2}} L^{p}$ with norm

$$
\|F\|_{r, p}=\left\|(1-\mathcal{L})^{r / 2} F\right\|_{p}, \quad F \in D^{r, p}, r>0,1 \leq p<\infty .
$$

Let $\mathcal{C}$ denote the space of continuous functions from $[0,1]$ to $\mathbb{R}^{d}$ endowed with the usual norm $\|f\|:=\sup _{0 \leq t \leq 1}|f(t)|$. We denote $\mathcal{C}_{x}:=\{f \in \mathcal{C}: f(0)=x\}$ and denote

$$
\mathcal{H}^{d}:=\left\{f \in \mathcal{C}_{0}: f(t)=\int_{0}^{t} \dot{f}(s) d s,\|f\|_{\mathcal{H}^{d}}^{2}:=\int_{0}^{1}|\dot{f}(t)|^{2} d t<\infty\right\} .
$$

It is clear that $\mathcal{H}^{d}$ is a Hilbert space with scalar product

$$
\left\langle r_{1}, r_{2}\right\rangle_{\mathcal{H}^{d}}=\int_{0}^{1}\left(\dot{r}_{1}(s), \dot{r}_{2}(s)\right) d s .
$$

Let the function $I: B \rightarrow[0, \infty]$ be defined by $I(f)=\frac{1}{2}\|f\|_{\mathcal{H}^{d}}^{2}$, if $f \in \mathcal{H}^{d}$ and let $I(f)=+\infty$, otherwise. Let $\mu$ be the Wiener measure. Then $\left(\mathcal{C}_{0}, \mathcal{H}^{d}, \mu\right)$ is an

Received by the editors December 25, 2009.

2010 Mathematics Subject Classification. Primary 60F15, 60F10, 60G17.

Key words and phrases. $C_{r, p}$-capacity, rate of local convergence, Hölder norm, large and small deviations.

The research is supported by the Science Research Foundations for the doctoral program of Guilin University of Electronic Technology under grant UF09007Y and by the Guangxi Natural Science Foundations under grant 2010GXNSB013049.

(C)2011 American Mathematical Society Reverts to public domain 28 years from publication 
abstract Wiener space. We will consider two Banach spaces as follows:

$$
\begin{aligned}
\mathcal{C}^{\alpha} & =\left\{f \in \mathcal{C}_{0}:\|f\|_{\alpha}=\sup _{s, t \in[0,1], s \neq t} \frac{|f(t)-f(s)|}{|t-s|^{\alpha}}<\infty\right\}, \\
\mathcal{C}^{\alpha, 0} & =\left\{f \in \mathcal{C}^{\alpha}: \lim _{\delta \rightarrow 0} \sup _{s, t \in[0,1], 0<|t-s|<\delta} \frac{|f(t)-f(s)|}{|t-s|^{\alpha}}=0\right\},
\end{aligned}
$$

where $0<\alpha<\frac{1}{2}$. Clearly, the space $\left(\mathcal{C}^{\alpha, 0}, \mathcal{H}^{d}, \mu\right)$ is also an abstract Wiener space; see, e.g., [1].

The capacity is a set function on $B$ with the property that it may take positive values even for $\mu$-null sets. For $r>0$ and $p>1$, the $(r, p)$-capacity is defined by

$$
C_{r, p}(O)=\inf \left\{\|F\|_{r, p}^{p}: F \geq 1, \mu-\text { a.s. on } O\right\}, \quad \text { for open sets } O \subset B,
$$

and for any set $A \subset B$,

$$
C_{r, p}(A)=\inf \left\{C_{r, p}(O): A \subset O \subset B, O \text { is open }\right\} .
$$

A large deviation principle for $C_{r, p}$ has been established by N. Yoshida [6]. The rate of clustering for Brownian motion in the Hölder norm was obtained by $\mathrm{P}$. Baldi and B. Roynette [2]. In this paper, our aim is to estimate the quasi sure local convergence rate for a Brownian motion in the Hölder norm. We also investigate the quasi sure local convergence rate for increments of a Brownian motion in the Hölder norm.

\section{Local functional Convergence Rate of Strassen type IN THE HÖLDER NORM}

The functional law of the iterated logarithm (LIL) of Strassen type in the Hölder norm was derived by P. Baldi, G. Ben Arous and G. Kerkyacharian in 1]. X. Chen and N. Balakrishnan obtained the functional LIL of Strassen type in the Hölder norm w.r.t. $C_{r, p}$-capacity 4 . We now present the local convergence rate of functional LIL of Strassen type for a Brownian motion in the Hölder norm with respect to $C_{r, p}$-capacity on an abstract Wiener space.

Suppose $w \in \mathcal{C}^{\alpha, 0}$ and let $K=\left\{f \in \mathcal{H}^{d}: I(f) \leq 1\right\}$. In [2, the authors proved that there exists a constant $k(\alpha)>0$ such that

$$
\lim _{\varepsilon \rightarrow 0} \varepsilon^{2 /(1-2 \alpha)} \log P\left\{\|w\|_{\alpha} \leq \varepsilon\right\}=-k(\alpha) .
$$

Moreover, for every $f \in K$ and $\gamma=(1-2 \alpha) / 2$,

$$
\lim _{\varepsilon \rightarrow 0} \varepsilon^{1 / \gamma} \log P\left(\left\|w-\frac{f}{\varepsilon^{1 /(2 \gamma)}}\right\|_{\alpha} \leq r \varepsilon\right)=-I(f)-\frac{k(\alpha)}{r^{1 / \gamma}} .
$$

Let us use the notation $L L(t):=\log \log t$ in the sequel. We state the main result of this section as follows.

Theorem 2.1. Let $0<\alpha<1, \gamma=\frac{1}{2}-\alpha$ and let $f \in K$. If $f$ satisfies $I(f)<1$, then we have

$$
\liminf _{t \rightarrow 0}\left(L L\left(t^{-1}\right)\right)^{1-\alpha}\left\|\frac{w(t \cdot)}{\sqrt{t L L\left(t^{-1}\right)}}-f\right\|_{\alpha}=\left(\frac{k(\alpha)}{1-I(f)}\right)^{\gamma}, \quad C_{r, p}-q . s .
$$

where $k(\alpha)>0$ is defined as in (2.1). 
The theorem follows from Lemmas 2.6] and 2.9, which we will state and prove in the rest of this section.

Lemma 2.2. Let $k$ be a natural number and let $q_{1}, q_{2} \in(1, \infty)$ satisfy $\frac{1}{p}=\frac{1}{q_{1}}+\frac{1}{q_{2}}$. Then, there exists a constant $c=c\left(k, p, q_{1}, q_{2}\right)>0$ such that for any $\delta \in(0,1)$,

$$
\begin{aligned}
& C_{k, p}\left(\bigcap_{i=1}^{n}\left\{z: a_{i}<\tilde{F}_{i}(z)<b_{i}\right\}\right)^{1 / p} \\
& \quad \leq c\left(\frac{n}{\delta}\right)^{k}\left(1+\max _{1 \leq i \leq n}\left\|F_{i}\right\|_{k, k q_{1}}\right)^{k} \mu\left(\bigcap_{i=1}^{n}\left\{a_{i}-\delta<F_{i}(z)<b_{i}+\delta\right\}\right)^{1 / q_{2}}
\end{aligned}
$$

for any $F_{i} \in D^{k, k q_{1}}$ and $-\infty<a_{i}<b_{i}<\infty$ holds. Here $\tilde{F}_{i}$ denotes a quasi continuous modification of $F_{i}$.

Proof. Set

$$
\chi(z)= \begin{cases}1, & \text { if } \bigcap_{i=1}^{n}\left\{z: a_{i}-\delta<F_{i}(z)<b_{i}+\delta\right\} \\ 0, & \text { otherwise. }\end{cases}
$$

We see that for $m \leq k, n_{1}+\cdots+n_{m}=l \leq k, i_{1}, \cdots, i_{m} \leq n$,

$$
\begin{aligned}
\left\|\chi D^{n_{1}} F_{i_{1}} \otimes \cdots \otimes D^{n_{m}} F_{i_{m}}\right\|_{p} & \leq\|\chi\|_{q_{2}} \prod_{a=1}^{m}\left\|D^{n_{a}} F_{i_{a}}\right\|_{m q_{1}} \\
& \leq c_{k, q_{1}}\left(\max _{1 \leq i \leq n}\left\|F_{i}\right\|_{k, m q_{1}}+1\right)^{m}\|\chi\|_{q_{2}} .
\end{aligned}
$$

The rest of the proof is the same as that of Proposition 3.1 in [6].

Lemma 2.3. Let $k, p, q_{1}, q_{2}$ be defined as in Lemma 2.2. For any $f \in K$ and $\varepsilon>0$, we set

$F_{\varepsilon}^{(i)}(w)=\left\|\varepsilon\left(\frac{w\left(t_{i}+\cdot h_{i}\right)-w\left(t_{i}\right)}{\sqrt{h_{i}}}\right)-f\right\|_{\alpha}, \quad 0 \leq t_{i}<\infty, h_{i}>0, i=1,2, \cdots, n$.

Then there exists a constant $c=c\left(k, p, q_{1}, f, d\right)>0$ such that for any $\delta \in(0,1], \varepsilon \in$ $(0,1]$, we have

$$
\begin{aligned}
& C_{k, p}\left(\bigcap_{i=1}^{n}\left\{z: a_{i}<F_{\varepsilon}^{(i)}(z)<b_{i}\right\}\right)^{\frac{1}{p}} \\
& \quad \leq c \delta^{-2 k^{2}-k} n^{k} \mu\left(\bigcap_{i=1}^{n}\left\{z: a_{i}-\delta<F_{\varepsilon}^{(i)}(z)<b_{i}+\delta\right\}\right)^{\frac{1}{q_{2}}} .
\end{aligned}
$$

Proof. Let $F: B \rightarrow R$ be a Borel function which represents an element $\bar{F}$ in $L^{p}$. We set

$$
\left(T_{t} F\right)(z)=\int_{B} F\left(e^{-t} z+\sqrt{1-e^{-2 t}} y\right) \mu(d y)
$$

Then $T_{t} F$ is a quasi continuous version of $e^{t \mathcal{L}} \bar{F}$. Here $\left(e^{t \mathcal{L}}\right)_{t>0}$ is the OrnsteinUhlenbeck semigroup acting on $L^{p}$. 
For any $q_{1}>0, F_{\varepsilon}^{(i)} \in L^{q_{1}}, i=1,2, \cdots, n, \varepsilon>0$, we know that $T_{t} F_{\varepsilon}^{(i)}$ represents an element in $\bigcap_{l=1}^{\infty} D^{l, k q_{1}}$. We also have the following estimate:

$$
\left\|T_{t} F_{\varepsilon}^{(i)}\right\|_{2 k, k q_{1}}=e^{t}\left\|(1-\mathcal{L})^{k} e^{-t(1-\mathcal{L})} F_{\varepsilon}^{(i)}\right\|_{k q_{1}} \leq c_{1} e^{t} t^{-k}\left\|F_{\varepsilon}^{(i)}\right\|_{k q_{1}}
$$

where $c_{1}=c_{1}\left(k, q_{1}\right)>0$. By the definition of $F_{\varepsilon}^{(i)}$, we have

$$
\begin{aligned}
F_{\varepsilon}^{(i)}\left(e^{-t} z+\sqrt{1-e^{-2 t}} y\right) \leq & e^{-t} F_{\varepsilon}^{(i)}(z)+\sqrt{1-e^{-2 t}} F_{\varepsilon}^{(i)}(y) \\
& +\left|e^{-t}+\sqrt{1-e^{-2 t}}-1\right| \cdot\|f\|_{\alpha} .
\end{aligned}
$$

Therefore, we deduce that

$$
\begin{aligned}
& T_{t} F_{\varepsilon}^{(i)}(z) \\
= & \int_{\mathcal{C}^{\alpha, 0}} F_{\varepsilon}^{(i)}\left(e^{-t} z+\sqrt{1-e^{-2 t}} y\right) \mu(d y) \\
\leq & e^{-t} F_{\varepsilon}^{(i)}(z)+\sqrt{1-e^{-2 t}} \int_{\mathcal{C}^{\alpha, 0}} F_{\varepsilon}^{(i)}(y) \mu(d y)+\left|e^{-t}+\sqrt{1-e^{-2 t}}-1\right| \cdot\|f\|_{\alpha} \\
\leq & e^{-t} F_{\varepsilon}^{(i)}(z)+e^{t} \sqrt{1-e^{-2 t}} \int_{\mathcal{C}^{\alpha, 0}} F_{\varepsilon}^{(i)}(y) \mu(d y)+e^{t}\left|e^{-t}+\sqrt{1-e^{-2 t}}-1\right| \cdot\|f\|_{\alpha} \\
= & e^{-t} F_{\varepsilon}^{(i)}(z)+C_{t},
\end{aligned}
$$

where $C_{t}=e^{t} \sqrt{1-e^{-2 t}} \int_{\mathcal{C}^{\alpha, 0}} F_{\varepsilon}^{(i)}(y) \mu(d y)+e^{t}\left|e^{-t}+\sqrt{1-e^{-2 t}}-1\right|\|f\|_{\alpha}$. Similarly, we can also derive $F_{\varepsilon}^{(i)}(z) \leq e^{t} T_{t} F_{\varepsilon}^{(i)}(z)+C_{t}$. By applying Lemma 2.2, similarly to the proof of Lemma 2.3 in [7, we complete the proof of Lemma 2.3.

Lemma 2.4. Let the constant $k(\alpha)>0$ be defined as in (2.1). For any $f \in K$, $\tau>0, h>0$, we have

$$
\begin{aligned}
& \lim _{\varepsilon \rightarrow 0} \varepsilon^{1 / \gamma} \log C_{r, p}\left(\left\|\frac{w(h \cdot)}{\sqrt{h}}-\frac{f}{\varepsilon^{1 /(2 \gamma)}}\right\|_{\alpha} \leq \varepsilon \tau\right) \\
= & \lim _{\varepsilon \rightarrow 0} \varepsilon^{1 / \gamma} \log \mu\left(\left\|\frac{w(h \cdot)}{\sqrt{h}}-\frac{f}{\varepsilon^{1 /(2 \gamma)}}\right\|_{\alpha} \leq \varepsilon \tau\right) \\
= & -\frac{k(\alpha)}{\tau^{1 / \gamma}}-I(f) .
\end{aligned}
$$

Proof. Let $k, p, q_{1}, q_{2}$ be as in Lemma 2.2. Since $C_{r, p}(\cdot) \geq \mu(\cdot)$, it suffices to prove that

$$
\begin{aligned}
& \lim _{\varepsilon \rightarrow 0} \varepsilon^{1 / \gamma} \log C_{r, p}\left(\left\|\frac{w(h \cdot)}{\sqrt{h}}-\frac{f}{\varepsilon^{1 /(2 \gamma)}}\right\|_{\alpha} \leq \varepsilon \tau\right) \\
\leq & \lim _{\varepsilon \rightarrow 0} \varepsilon^{1 / \gamma} \log \mu\left(\left\|\frac{w(h \cdot)}{\sqrt{h}}-\frac{f}{\varepsilon^{1 /(2 \gamma)}}\right\|_{\alpha} \leq \varepsilon \tau\right) .
\end{aligned}
$$


Set $k=[r]+1$. By Lemma 2.3, for any $1>\delta>0$ and $c_{0}>0$, we have

$$
\begin{aligned}
& C_{r, p}\left(\left\|\frac{w(h \cdot)}{\sqrt{h}}-\frac{f}{\varepsilon^{1 /(2 \gamma)}}\right\|_{\alpha} \leq \varepsilon \tau\right)^{1 / p} \\
= & C_{r, p}\left(\left\|\varepsilon^{1 /(2 \gamma)} \frac{w(h \cdot)}{\sqrt{h}}-f\right\|_{\alpha} \leq \varepsilon^{1 /(2 \gamma)+1} \tau\right)^{1 / p} \\
\leq & c_{0}\left(\varepsilon^{1 /(2 \gamma)+1} \delta\right)^{-2 k^{2}-k} \mu\left(\left\|\varepsilon^{1 /(2 \gamma)} \frac{w(h \cdot)}{\sqrt{h}}-f\right\|_{\alpha} \leq \varepsilon^{1 /(2 \gamma)+1}(\tau+\delta)\right)^{1 / q_{2}} \\
= & c_{0}\left(\varepsilon^{1 /(2 \gamma)+1} \delta\right)^{-2 k^{2}-k} \mu\left(\left\|w(\cdot)-\frac{f}{\varepsilon^{1 /(2 \gamma)}}\right\|_{\alpha} \leq \varepsilon(\tau+\delta)\right)^{1 / q_{2}} .
\end{aligned}
$$

By (2.2), we have

$$
\begin{aligned}
& \lim _{\varepsilon \rightarrow 0} \varepsilon^{1 / \gamma} \log C_{r, p}\left(\left\|\frac{w(h \cdot)}{\sqrt{h}}-\frac{f}{\varepsilon^{1 /(2 \gamma)}}\right\|_{\alpha} \leq \varepsilon \tau\right) \\
\leq & \frac{p}{q_{2}} \lim _{\varepsilon \rightarrow 0} \varepsilon^{1 / \gamma} \log \mu\left(\left\|w(\cdot)-\frac{f}{\varepsilon^{1 /(2 \gamma)}}\right\|_{\alpha} \leq \varepsilon(\tau+\delta)\right) \\
= & \frac{p}{q_{2}}\left(-k(\alpha)(\tau+\delta)^{-\frac{1}{\gamma}}-I(f)\right) .
\end{aligned}
$$

Letting $\delta \rightarrow 0$ and $q_{2} \rightarrow p$, we end the proof of Lemma 2.4

Lemma 2.5. For $f \in K$ with $I(f)<1$, we have

$$
\liminf _{n \rightarrow \infty}\left(L L\left(t_{n}^{-1}\right)\right)^{1-\alpha}\left\|\frac{w\left(t_{n} \cdot\right)}{\sqrt{t_{n} L L\left(t_{n}^{-1}\right)}}-f\right\|_{\alpha} \geq\left(\frac{k(\alpha)}{1-I(f)}\right)^{\gamma}, \quad C_{r, p}-q . s .
$$

where $t_{n}=\exp \left(-\frac{n}{(\log n)^{a}}\right), a>0$.

Proof. For any $\varepsilon \in(0,1), f \in K$, since $I(f)<1$, choose $\delta_{1}>0$ such that $\eta_{0}=$ $I(f)+\frac{1-I(f)}{(1-\varepsilon)^{1 / \gamma}}-\delta_{1}>1$. By Lemma 2.4, for $n$ large enough, we have

$$
\begin{aligned}
& C_{r, p}\left(\left(L L\left(t_{n}^{-1}\right)\right)^{1-\alpha}\left\|\frac{w\left(t_{n} \cdot\right)}{\sqrt{t_{n} L L\left(t_{n}^{-1}\right)}}-f\right\|_{\alpha} \leq(1-\varepsilon)\left(\frac{k(\alpha)}{1-I(f)}\right)^{\gamma}\right) \\
= & C_{r, p}\left(\left\|\frac{w\left(t_{n} \cdot\right)}{\sqrt{t_{n}}}-\left(L L\left(t_{n}^{-1}\right)\right)^{1 / 2} f\right\|_{\alpha} \leq\left(L L\left(t_{n}^{-1}\right)\right)^{-\frac{1}{2}+\alpha}(1-\varepsilon)\left(\frac{k(\alpha)}{1-I(f)}\right)^{\gamma}\right) \\
\leq & \exp \left\{\left(L L\left(t_{n}^{-1}\right)\right)\left(-\frac{1-I(f)}{(1-\varepsilon)^{1 / \gamma}}-I(f)+\delta_{1}\right)\right\}=\left(\frac{1}{\log t_{n}^{-1}}\right)^{\eta_{0}} .
\end{aligned}
$$

By Borel-Cantelli's Lemma, we have

$$
\liminf _{n \rightarrow \infty}\left(L L\left(t_{n}^{-1}\right)\right)^{1-\alpha}\left\|\frac{w\left(t_{n} \cdot\right)}{\sqrt{t_{n} L L\left(t_{n}^{-1}\right)}}-f\right\|_{\alpha} \geq\left(\frac{k(\alpha)}{1-I(f)}\right)^{\gamma}, \quad C_{r, p}-q . s .
$$


Lemma 2.6. For any $f \in K$ with $I(f)<1$, we have

$$
\liminf _{t \rightarrow 0}\left(L L\left(t^{-1}\right)\right)^{1-\alpha}\left\|\frac{w(t \cdot)}{\sqrt{t L L\left(t^{-1}\right)}}-f\right\|_{\alpha} \geq\left(\frac{k(\alpha)}{1-I(f)}\right)^{\gamma}, \quad C_{r, p}-q . s .
$$

Proof. Let $\psi_{t}(s)=\frac{w(t s)}{\sqrt{t L L\left(t^{-1}\right)}}, s \in[0,1], t \in[0,1]$. Let $t_{n}$ be as in Lemma 2.5 For $t_{n+1}<t \leq t_{n}$, we set $X(t)=\left(L L\left(t^{-1}\right)\right)^{1-\alpha}\left\|\psi_{t}(\cdot)-f\right\|_{\alpha}$ and $X_{n}=\inf _{t_{n+1}<t \leq t_{n}} X(t)$. For any $\varepsilon>0$, by the definition of the infimum, there exists $T_{n} \in\left(t_{n+1}, t_{n}\right]$ such that $X_{n} \geq X\left(T_{n}\right)-\varepsilon$. For any $u, v \in[0,1]$, let $x=\frac{u t_{n+1}}{T_{n}}, y=\frac{v t_{n+1}}{T_{n}}$; then $0 \leq x, y \leq \frac{t_{n+1}}{T_{n}} \leq 1$. We have

$$
\begin{aligned}
& \left\|\psi_{t_{n+1}}(\cdot)-f\right\|_{\alpha} \\
= & \sup _{0 \leq u<v \leq 1} \frac{\left|\left(\psi_{t_{n+1}}(u)-f(u)\right)-\left(\psi_{t_{n+1}}(v)-f(v)\right)\right|}{|u-v|^{\alpha}} \\
\leq & \sup _{0 \leq x<y \leq \frac{t_{n+1}}{T_{n}}} \frac{\left|\left(\psi_{t_{n+1}}\left(\frac{T_{n} x}{t_{n+1}}\right)-f\left(\frac{T_{n} x}{t_{n+1}}\right)\right)-\left(\psi_{t_{n+1}}\left(\frac{T_{n} y}{t_{n+1}}\right)-f\left(\frac{T_{n} y}{t_{n+1}}\right)\right)\right|}{|x-y|^{\alpha}} \\
\leq & \left\|\gamma_{n} \psi_{T_{n}}(\cdot)-f\left(\frac{T_{n}}{t_{n+1}} \cdot\right)\right\|_{\alpha} \\
\leq & \gamma_{n}\left\|\psi_{T_{n}}(\cdot)-f(\cdot)\right\|_{\alpha}+\left|\gamma_{n}-1\right|\|f(\cdot)\|_{\alpha}+\left\|f(\cdot)-f\left(\frac{T_{n}}{t_{n+1}} \cdot\right)\right\|_{\alpha},
\end{aligned}
$$

where we use the notation $\gamma_{n}=\frac{\sqrt{T_{n} L L\left(T_{n}^{-1}\right)}}{\sqrt{t_{n+1} L L\left(t_{n+1}^{-1}\right)}}$.

By (2.6), we have

$X\left(T_{n}\right) \geq \tilde{\gamma}_{n}\left(L L t_{n+1}^{-1}\right)^{1-\alpha}\left(\left\|\psi_{t_{n+1}}(\cdot)-f\right\|_{\alpha}-\left|\gamma_{n}-1\right|\|f(\cdot)\|_{\alpha}-\left\|f(\cdot)-f\left(\frac{T_{n}}{t_{n+1}} \cdot\right)\right\|_{\alpha}\right)$,

where $\tilde{\gamma}_{n}=\sqrt{\frac{t_{n}}{t_{n+1}}}\left(\frac{L L\left(t_{n}^{-1}\right)}{L L\left(t_{n+1}^{-1}\right)}\right)^{\frac{1}{2}-\alpha}$.

The inequality $\exp (-x) \geq 1-x$ yields

$$
\frac{t_{n+1}}{t_{n}} \geq 1-\frac{n+1}{(\log (n+1))^{a}}+\frac{n}{(\log n)^{a}} .
$$

From the inequality above we have

$$
1-\frac{t_{n+1}}{t_{n}} \leq \frac{1}{(\log n)^{a}} .
$$

Similar to the proof of (5.3) in [2], we have

$$
\left\|f\left(\frac{T_{n}}{t_{n+1}} \cdot\right)-f(\cdot)\right\|_{\alpha} \leq 2\left(\frac{T_{n}}{t_{n+1}}-1\right)^{\frac{1}{2}-\alpha} \leq 2\left(\frac{t_{n}}{t_{n+1}}-1\right)^{\frac{1}{2}-\alpha} .
$$

Since $t_{n} / t_{n+1} \rightarrow 1$, as $n \rightarrow \infty$, for $n$ large enough, we have $t_{n} / t_{n+1}<2$. By (2.8) and (2.9), for $n$ large enough, we have

$$
\left\|f\left(\frac{T_{n}}{t_{n+1}} \cdot\right)-f(\cdot)\right\|_{\alpha} \leq 4\left(\frac{1}{(\log n)^{a}}\right)^{\frac{1}{2}-\alpha} .
$$


Noting the above, for $n$ large enough, we have

$$
\left|\frac{\left(T_{n} L L\left(T_{n}^{-1}\right)\right)^{1 / 2}}{\left(t_{n+1} L L\left(t_{n+1}^{-1}\right)\right)^{1 / 2}}-1\right| \leq\left|\frac{t_{n}}{t_{n+1}}-1\right| \leq \frac{2}{(\log n)^{a}} .
$$

Choosing a suitable $a$, by (2.7), (2.10), (2.11) and Lemma 2.5, we have

$$
\liminf _{n \rightarrow \infty} X\left(T_{n}\right) \geq\left(\frac{k(\alpha)}{1-I(f)}\right)^{\gamma}, \quad C_{r, p}-q . s .
$$

Since

$$
\liminf _{t \rightarrow 0} X(t) \geq \liminf _{n \rightarrow \infty} X_{n} \geq \liminf _{n \rightarrow \infty} X\left(T_{n}\right)-\varepsilon
$$

we can conclude (2.5).

Lemma 2.7. For $u \geq 3$, we define

$$
\xi_{u}(t, w)=\frac{w\left(\frac{t}{u}\right)}{\sqrt{\frac{2}{u} \log \log u}}, \quad t \in[0,1], w \in \mathcal{C}^{\alpha, 0} .
$$

We have, for any $(r, p) \in(0, \infty) \times(1, \infty)$, for $C_{r, p}-q . s . w,\left\{\xi_{u}(\cdot, w), u \geq 3\right\}$ is relatively compact in $\mathcal{C}^{\alpha, 0}$, and the set of its limit points is a subset of $C:=\{f \in$ $\left.\mathcal{H}^{d}:\|f\|_{\mathcal{H}^{d}}^{2} \leq 1\right\}$ as $u \rightarrow \infty$.

Proof. Since $C$ is compact in $\mathcal{C}^{\alpha, 0}$, it is sufficient to prove that

$$
\lim _{u \rightarrow \infty}\left\|\xi_{u}(\cdot, w)-C\right\|_{\alpha}=0, \quad C_{r, p}-q . s .
$$

Noting that

$$
\begin{aligned}
& \mu\left(\left\|\frac{w(\dot{\bar{u}})}{\sqrt{\frac{2}{u} \log \log u}}-C\right\|_{\alpha} \geq \varepsilon\right) \\
= & \mu\left(\left\|\frac{1}{\sqrt{2 \log \log u}} w(\cdot)-C\right\|_{\alpha} \geq \varepsilon\right)=\mu\left(\frac{1}{\sqrt{2 \log \log u}} w \in A\right),
\end{aligned}
$$

where $A=\left\{f \in \mathcal{C}^{\alpha, 0}:\|f(\cdot)-C\|_{\alpha} \geq \varepsilon\right\}$, it is clear that the set $A$ is closed and for any $f \in A$,

$$
\int_{0}^{1}|\dot{f}(s)|^{2} d s>1 .
$$

Accordingly, there exists $\delta>0$ such that $\inf _{f \in A} I(f)>\frac{1}{2}+\frac{\delta}{2}$. For $\theta>1$, and letting $u_{n}=\theta^{n}$, for $n$ large enough, by Theorem 2.1 in [4, we have

$$
C_{r, p}\left(\left\|\frac{w\left(\dot{\overline{u_{n}}}\right)}{\sqrt{\frac{2}{u_{n}} \log \log u_{n}}}-C\right\|_{\alpha} \geq \varepsilon\right) \leq \exp \left(-(1+\delta) \log \log u_{n}\right) .
$$

By Borel-Cantelli's Lemma,

$$
\limsup _{n \rightarrow \infty}\left\|\xi_{u_{n}}(\cdot, w)-C\right\|_{\alpha}=0, \quad C_{r, p}-q . s .
$$

Thus, by Lemma 3.1 in 4], we have

$$
\lim _{u \rightarrow \infty}\left\|\xi_{u}(\cdot, w)-C\right\|_{\alpha}=0, \quad C_{r, p}-q . s .
$$


Lemma 2.8. Let $t_{n}=\frac{1}{n^{n}}$. For $\varphi \in K$ with $I(\varphi)<1$, we have

$$
\liminf _{n \rightarrow \infty}\left(L L\left(t_{n}^{-1}\right)\right)^{1-\alpha}\left\|\frac{w\left(t_{n} \cdot\right)}{\sqrt{t_{n} L L\left(t_{n}^{-1}\right)}}-\varphi\right\|_{\alpha} \leq\left(\frac{k(\alpha)}{1-I(\varphi)}\right)^{\gamma}, \quad C_{r, p}-q . s .
$$

Proof. We write $g_{n}(s)=\frac{w\left(t_{n} s\right)}{\sqrt{t_{n} L L\left(t_{n}^{-1}\right)}}-\varphi(s)$,

$$
\begin{aligned}
\left\|g_{n}(\cdot)\right\|_{\alpha} & =\sup _{0 \leq t<s \leq 1} \frac{\left|g_{n}(s)-g_{n}(t)\right|}{|s-t|^{\alpha}} \\
& \leq \sup _{0 \leq t<s \leq \frac{t_{n+1}}{t_{n}}} \frac{\left|g_{n}(s)-g_{n}(t)\right|}{|s-t|^{\alpha}}+\sup _{\frac{t_{n+1}}{t_{n}} \leq t<s \leq 1} \frac{\left|g_{n}(s)-g_{n}(t)\right|}{|s-t|^{\alpha}} \\
(2.12) \quad & \leq\left(\frac{t_{n}}{t_{n+1}}\right)^{\alpha}\left\|\frac{w\left(t_{n+1} \cdot\right)}{\sqrt{t_{n} L L\left(t_{n}^{-1}\right)}}-\varphi\left(\frac{t_{n+1}}{t_{n}} \cdot\right)\right\|_{\alpha}+\sup _{\frac{t_{n+1}}{t_{n}} \leq t<s \leq 1} \frac{\left|g_{n}(s)-g_{n}(t)\right|}{|s-t|^{\alpha}} .
\end{aligned}
$$

In the following, we prove that

$$
\limsup _{n \rightarrow \infty}\left(L L\left(t_{n}^{-1}\right)\right)^{1-\alpha}\left(\frac{t_{n}}{t_{n+1}}\right)^{\alpha}\left\|\frac{w\left(t_{n+1} \cdot\right)}{\sqrt{t_{n} L L\left(t_{n}^{-1}\right)}}-\varphi\left(\frac{t_{n+1}}{t_{n}} \cdot\right)\right\|_{\alpha}=0, \quad C_{r, p}-\text { q.s. }
$$

In fact

$$
\begin{aligned}
\left\|g_{n}\left(\frac{t_{n+1}}{t_{n}}\right)\right\|_{\alpha} & \leq\left\|\frac{w\left(t_{n+1} \cdot\right)}{\left(t_{n} L L\left(t_{n}^{-1}\right)\right)^{1 / 2}}\right\|_{\alpha}+\left\|\varphi\left(\frac{t_{n+1}}{t_{n}} \cdot\right)\right\|_{\alpha} \\
& =\frac{\left(t_{n+1} L L\left(t_{n+1}^{-1}\right)\right)^{1 / 2}}{\left(t_{n} L L\left(t_{n}^{-1}\right)\right)^{1 / 2}}\left\|\frac{w\left(t_{n+1} \cdot\right)}{\sqrt{t_{n+1} L L\left(t_{n+1}^{-1}\right)}}\right\|_{\alpha}+\left\|\varphi\left(\frac{t_{n+1}}{t_{n}} \cdot\right)\right\|_{\alpha} .
\end{aligned}
$$

Since $\left\|\frac{w\left(t_{n+1} \cdot\right)}{\sqrt{t_{n+1} L L\left(t_{n+1}^{-1}\right)}}\right\|_{\alpha}$ is bounded by local Strassen's law (see Lemma 2.7) and

$$
\left(L L\left(t_{n}^{-1}\right)\right)^{1-\alpha}\left(\frac{t_{n}}{t_{n+1}}\right)^{\alpha} \frac{\left(t_{n+1} L L\left(t_{n+1}^{-1}\right)\right)^{1 / 2}}{\left(t_{n} L L\left(t_{n}^{-1}\right)\right)^{1 / 2}} \rightarrow 0, \text { as } n \rightarrow \infty,
$$

we get

$$
\left(L L\left(t_{n}^{-1}\right)\right)^{1-\alpha}\left(\frac{t_{n}}{t_{n+1}}\right)^{\alpha} \frac{\left(t_{n+1} L L\left(t_{n+1}^{-1}\right)\right)^{1 / 2}}{\left(t_{n} L L\left(t_{n}^{-1}\right)\right)^{1 / 2}}\left\|\frac{w\left(t_{n+1} \cdot\right)}{\sqrt{t_{n+1} L L\left(t_{n+1}^{-1}\right)}}\right\|_{\alpha} \rightarrow 0, \text { as } n \rightarrow \infty .
$$

Since $f \in K$, for $\gamma \leq 1$, we have $\|f(\gamma \cdot)\|_{\alpha} \leq\|f(\gamma \cdot)\|_{\mathcal{H}^{d}} \leq \gamma^{1 / 2}\|f\|_{\mathcal{H}^{d}}$ (see the proof of Theorem 5.1 in [2]). Therefore we deduce that

$$
\begin{aligned}
& \left(L L\left(t_{n}^{-1}\right)\right)^{1-\alpha}\left(\frac{t_{n}}{t_{n+1}}\right)^{\alpha}\left\|\varphi\left(\frac{t_{n+1}}{t_{n}} \cdot\right)\right\|_{\alpha} \\
\leq & 2\left(L L\left(t_{n}^{-1}\right)\right)^{1-\alpha}\left(\frac{t_{n}}{t_{n+1}}\right)^{\alpha} \sqrt{\frac{t_{n+1}}{t_{n}}} \\
= & 2(\log n+L L(n))^{1-\alpha}\left(\frac{n^{n}}{(n+1)^{(n+1)}}\right)^{1 / 2-\alpha} \rightarrow 0, \text { as } n \rightarrow \infty .
\end{aligned}
$$


So we obtain (2.13).

We set $w_{n}(s)=\frac{w\left(t_{n} s\right)-w\left(t_{n+1}\right)}{\sqrt{t_{n} L L\left(t_{n}^{-1}\right)}}-\varphi(s)$ for convenience. We now prove that

$$
\liminf _{n \rightarrow \infty}\left(L L\left(t_{n}^{-1}\right)\right)^{1-\alpha} \sup _{\frac{t_{n+1}}{t_{n}} \leq t<s \leq 1} \frac{\left|w_{n}(s)-w_{n}(t)\right|}{|s-t|^{\alpha}} \leq\left(\frac{k(\alpha)}{1-I(\varphi)}\right)^{\gamma}, \quad C_{r, p}-q . s .
$$

In order to complete the proof, we set

$$
\begin{aligned}
& \tilde{w}_{n}(s)=\frac{w\left(\left(t_{n}-t_{n+1}\right) s+t_{n+1}\right)-w\left(t_{n+1}\right)}{\sqrt{t_{n}-t_{n+1}}}, \quad s \geq 0, \\
& f\left(s_{1}\right)=\left(\frac{t_{n}}{t_{n}-t_{n+1}}\right)^{\frac{1}{2}}\left(\varphi\left(\frac{t_{n+1}+s_{1}\left(t_{n}-t_{n+1}\right)}{t_{n}}\right)-\varphi\left(\frac{t_{n+1}}{t_{n}}\right)\right), \quad s_{1} \in[0,1] .
\end{aligned}
$$

Then $\tilde{w}_{n}=\left\{\tilde{w}_{n}(s): s \geq 0\right\}$ is again a standard Brownian motion and $f \in K$ with $I(f) \leq I(\varphi)$. It is clear that $\frac{t_{n}}{t_{n}-t_{n+1}} \rightarrow 1$, as $n \rightarrow \infty$. For any $\varepsilon>0$, choose $\delta>0$ such that $\sigma:=\frac{1-I(f)}{(1+\varepsilon)^{1 / \gamma}}+I(f)+\delta<1$. By Lemma 2.3.

$$
\begin{aligned}
& C_{r, p}\left(\bigcap_{n=n_{0}}^{N}\left(L L\left(t_{n}^{-1}\right)^{1-\alpha} \sup _{\frac{t_{n+1}}{t_{n}} \leq t<s \leq 1} \frac{\left|w_{n}(s)-w_{n}(t)\right|}{|s-t|^{\alpha}} \geq\left(\frac{k(\alpha)}{1-I(\varphi)}\right)^{\gamma}(1+2 \varepsilon)\right)\right)^{\frac{1}{p}} \\
& \quad=C_{r, p}\left\{\bigcap_{n=n_{0}}^{N}\left(W^{\alpha}(n) \geq\left(\frac{k(\alpha)}{1-I(\varphi)}\right)^{\gamma} \frac{1+2 \varepsilon}{\left(L L\left(t_{n}^{-1}\right)\right)^{\frac{1}{2}-\alpha}}\right)\right\}^{1 / p} \\
& \quad \leq c N^{k}\left(L L\left(t_{N}^{-1}\right)^{\frac{1}{2}-\alpha}\right)^{2 k^{2}+k} \mu\left\{\bigcap_{n=n_{0}}^{N} W^{\alpha}(n) \geq\left(\frac{k(\alpha)}{1-I(f)}\right)^{\gamma} \frac{(1+\varepsilon)}{\left(L L\left(t_{n}^{-1}\right)\right)^{\frac{1}{2}-\alpha}}\right\}^{1 / q_{2}},
\end{aligned}
$$

where $W^{\alpha}(n)=\left(\frac{t_{n}}{t_{n}-t_{n+1}}\right)^{\alpha-\frac{1}{2}}\left\|\tilde{w}_{n}(\cdot)-\sqrt{L L\left(t_{n}^{-1}\right)} f(\cdot)\right\|_{\alpha}$. For $n$ large enough, by a small deviation,

$\mu\left\{\bigcap_{n=n_{0}}^{N} W^{\alpha}(n) \geq\left(\frac{k(\alpha)}{1-I(f)}\right)^{\gamma} \frac{(1+\varepsilon)}{\left(L L\left(t_{n}^{-1}\right)\right)^{\frac{1}{2}-\alpha}}\right\}^{1 / q_{2}} \leq \prod_{n=n_{0}}^{N}\left(1-\exp \left(-\sigma L L\left(t_{n}^{-1}\right)\right)\right)^{1 / q_{2}}$.

We further deduce that

$$
\begin{aligned}
& C_{r, p}\left(\bigcap_{n=n_{0}}^{N}\left(L L\left(t_{n}^{-1}\right)^{1-\alpha} \sup _{\frac{t_{n+1}}{t_{n}} \leq t<s \leq 1} \frac{\left|w_{n}(s)-w_{n}(t)\right|}{|s-t|^{\alpha}} \geq\left(\frac{k(\alpha)}{1-I(\varphi)}\right)^{\gamma}(1+2 \varepsilon)\right)\right)^{\frac{1}{p}} \\
& \quad \leq c N^{k}\left(L L\left(t_{N}^{-1}\right)^{\frac{1}{2}-\alpha}\right)^{2 k^{2}+k} \exp \left(-\frac{1}{q_{2}} \sum_{n=n_{0}+1}^{N+1}(n \log n)^{-\sigma}\right) \\
& \leq c_{0} N^{k}\left((\log N)^{\frac{1}{2}-\alpha}\right)^{2 k^{2}+k} \exp \left(-\frac{1}{q_{2}} \sum_{n=n_{0}+1}^{N+1}(n \log n)^{-\sigma}\right) \rightarrow 0 \quad(N \rightarrow \infty) .
\end{aligned}
$$


Therefore

$C_{r, p}\left\{\bigcup_{l=1}^{\infty} \bigcap_{n=l}^{\infty}\left(L L\left(t_{n}^{-1}\right)^{1-\alpha} \sup _{\frac{t_{n+1}}{t_{n}} \leq t<s \leq 1} \frac{\left|w_{n}(s)-w_{n}(t)\right|}{|s-t|^{\alpha}} \geq\left(\frac{k(\alpha)}{1-I(\varphi)}\right)^{\gamma}(1+2 \varepsilon)\right)\right\}=0$,

and we can conclude (2.14).

By Lemma 2.8, we can easily get

Lemma 2.9. For $\varphi \in K$ with $I(\varphi)<1$, we have

$$
\liminf _{t \rightarrow 0} L L\left(t^{-1}\right)^{1-\alpha}\left\|\frac{w(t \cdot)}{\sqrt{t L L\left(t^{-1}\right)}}-\varphi\right\|_{\alpha} \leq\left(\frac{k(\alpha)}{1-I(\varphi)}\right)^{\gamma}, \quad C_{r, p}-q . s .
$$

3. The local convergence rate for increments of a Brownian motion IN THE HÖLDER NORM

In previous sections, we get a quasi sure local functional convergence rate for a Brownian motion in the Hölder norm. In this section, we investigate the local convergence rate for increments of a Brownian motion in the Hölder norm with respect to $C_{r, p}$-capacity. We have the following theorem.

Theorem 3.1. Let $a_{u}$ be a non-decreasing function satisfying (1) $0<a_{u} \leq u<1$ and (2) $u / a_{u}$ being a non-increasing function. Set

$$
\ell_{u}=\log \frac{u \log u^{-1}}{a_{u}} \text { and } \beta_{u}=\left(a_{u} \ell_{u}\right)^{-1 / 2} .
$$

Let $\left(\mathcal{C}^{\alpha, 0}, \mathcal{H}^{d}, \mu\right)$ be an abstract Wiener space. For $w \in \mathcal{C}^{\alpha, 0}$, we denote

$$
\Delta(t, u)(s)=w\left(u t+a_{u} s\right)-w(u t), \quad s \in[0,1], t \in\left[0,1-\frac{a_{u}}{u}\right] .
$$

We have

$$
\liminf _{u \rightarrow 0} \ell_{u}^{1-\alpha} \inf _{t \in\left[0,1-\frac{a_{u}}{u}\right]}\left\|\beta_{u} \Delta(t, u)\right\|_{\alpha}=(k(\alpha))^{\gamma}, \quad C_{r, p}-q . s .
$$

Moreover, if condition (3) $\lim _{u \rightarrow 0} \frac{\log \left(u / a_{u}\right)}{L L\left(u^{-1}\right)}=\infty$ also holds, then

$$
\lim _{u \rightarrow 0} \ell_{u}^{1-\alpha} \inf _{t \in\left[0,1-\frac{a_{u}}{u}\right]}\left\|\beta_{u} \Delta(t, u)\right\|_{\alpha}=(k(\alpha))^{\gamma}, \quad C_{r, p}-q . s .
$$

Here $k(\alpha)$ is defined as in (2.1).

The proof of Theorem 3.1 is completed by the following lemmas:

Lemma 3.2. There exists a constant $k(\alpha)>0$, such that for any $f \in K, \tau>$ $0, h>0$, we have

$$
\begin{aligned}
& \lim _{\varepsilon \rightarrow 0} \varepsilon^{1 / \gamma} \log C_{r, p}\left(\left\|\frac{w(t+h \cdot)-w(t)}{\sqrt{h}}-\frac{f}{\varepsilon^{1 /(2 \gamma)}}\right\|_{\alpha} \leq \varepsilon \tau\right) \\
& =\lim _{\varepsilon \rightarrow 0} \varepsilon^{1 / \gamma} \log \mu\left(\left\|\frac{w(t+h \cdot)-w(t)}{\sqrt{h}}-\frac{f}{\varepsilon^{1 /(2 \gamma)}}\right\|_{\alpha} \leq \varepsilon \tau\right)=-\frac{k(\alpha)}{\tau^{1 / \gamma}}-I(f) .
\end{aligned}
$$

Proof. Similar to that of Lemma 2.4 .

Lemma 3.3. Let $k(\alpha)$ be defined as in (2.1). We have

$$
\liminf _{u \rightarrow 0} \ell_{u}^{1-\alpha} \inf _{t \in\left[0,1-\frac{a_{u}}{u}\right]}\left\|\beta_{u} \Delta(t, u)\right\|_{\alpha} \geq(k(\alpha))^{\gamma}, \quad C_{r, p}-q . s .
$$


Proof. Let $l(u)=a_{u}\left(\ell_{u}\right)^{-\frac{2}{1-2 \alpha}}$. For $\theta \in\left(1,(1-\varepsilon)^{-2}\right)$ we set $u_{n}=\theta^{-n}$ and $k_{n}=\left[\frac{u_{n}}{l\left(u_{n+1}\right)}\right]$ and $t_{i}=i l\left(u_{n+1}\right), i=0,1,2, \cdots, k_{n}$. Choose $\delta^{\prime}>0$ such that $\eta_{0}=\frac{1}{\left(\theta^{1 / 2}(1-\varepsilon)\right)^{1 / \gamma}}-\delta^{\prime}>1$. Then

$$
\begin{aligned}
& \min _{0<i \leq k_{n}}\left\|\beta_{u_{n}}\left(w\left(t_{i}+a_{u} \cdot\right)-w\left(t_{i}\right)\right)\right\|_{\alpha} \\
\leq & \max _{0 \leq i \leq k_{n}} \sup _{0 \leq S \leq l\left(u_{n+1}\right)}\left\|\beta_{u_{n}}\left(w\left(S+\left(t_{i}+a_{u} \cdot\right)\right)-w\left(t_{i}+a_{u} \cdot\right)\right)\right\|_{\alpha} \\
+ & \inf _{t \in\left[0, u_{n}-a_{u_{n+1}}\right]}\left\|\beta_{u_{n}}\left(w\left(t+a_{u} \cdot\right)-w(t)\right)\right\|_{\alpha} .
\end{aligned}
$$

For any $0<\varepsilon<1$, we have

$$
\begin{aligned}
& C_{r, p}\left(\left(\ell_{u_{n}}\right)^{1-\alpha} \min _{0 \leq i \leq k_{n}}\left\|\beta_{u_{n}}\left(w\left(t_{i}+a_{u} \cdot\right)-w\left(t_{i}\right)\right)\right\|_{\alpha} \leq(1-\varepsilon)(k(\alpha))^{\gamma}\right) \\
\leq & \sum_{0 \leq i \leq k_{n}} C_{r, p}\left(\left\|\beta_{u_{n}}\left(w\left(t_{i}+a_{u} \cdot\right)-w\left(t_{i}\right)\right)\right\|_{\alpha} \leq \frac{(1-\varepsilon)(k(\alpha))^{\gamma}}{\ell_{u_{n}}^{1-\alpha}}\right) \\
= & \sum_{0 \leq i \leq k_{n}} C_{r, p}\left(\left\|\frac{1}{\sqrt{\ell_{u_{n}}}} \frac{\left(w\left(t_{i}+a_{u} \cdot\right)-w\left(t_{i}\right)\right)}{\sqrt{a_{u}}}\right\|_{\alpha} \leq\left(\frac{a_{u_{n}}}{a_{u}}\right)^{1 / 2} \frac{(k(\alpha))^{\gamma}(1-\varepsilon)}{\ell_{u_{n}}^{1-\alpha}}\right) \\
\leq & \sum_{0 \leq i \leq k_{n}} C_{r, p}\left(\left\|\frac{1}{\sqrt{\ell_{u_{n}}}} \frac{\left(w\left(t_{i}+a_{u} \cdot\right)-w\left(t_{i}\right)\right)}{\sqrt{a_{u}}}\right\|_{\alpha} \leq \theta^{1 / 2} \frac{(k(\alpha))^{\gamma}(1-\varepsilon)}{\ell_{u_{n}}^{1-\alpha}}\right) .
\end{aligned}
$$

By Lemma 3.2, for $n$ large enough, we have

$$
\begin{aligned}
& \sum_{0 \leq i \leq k_{n}} C_{r, p}\left(\left\|\frac{1}{\sqrt{\ell_{u_{n}}}} \frac{\left(w\left(t_{i}+a_{u} \cdot\right)-w\left(t_{i}\right)\right)}{\sqrt{a_{u}}}\right\|_{\alpha} \leq \theta^{1 / 2} \frac{(k(\alpha))^{\gamma}(1-\varepsilon)}{\ell_{u_{n}}^{1-\alpha}}\right) \\
\leq & \left(1+k_{n}\right) \exp \left\{-\ell_{u_{n}} \eta_{0}\right\} \leq \frac{u_{n}+l\left(u_{n+1}\right)}{l\left(u_{n+1}\right)}\left(\frac{a_{u_{n}}}{u_{n} \log u_{n}^{-1}}\right)^{\eta_{0}} .
\end{aligned}
$$

Therefore, by Borel-Cantelli's Lemma,

$$
\liminf _{n \rightarrow \infty} \ell_{u_{n}}^{1-\alpha} \min _{0 \leq i \leq k_{n}}\left\|\beta_{u_{n}}\left(w\left(t_{i}+a_{u} \cdot\right)-w\left(t_{i}\right)\right)\right\|_{\alpha} \geq(k(\alpha))^{\gamma}, \quad C_{r, p}-q . s .
$$

On the other hand, for any $\eta>0$,

$$
\begin{aligned}
& C_{r, p}\left\{\ell_{u_{n}}^{1-\alpha} \sup _{0 \leq i \leq k_{n}} \sup _{0 \leq s \leq l\left(u_{n+1}\right)} \beta_{u_{n}}\left\|w\left(s+t_{i}+a_{u} \cdot\right)-w\left(t_{i}+a_{u} \cdot\right)\right\|_{\alpha} \geq \eta\right\} \\
= & C_{r, p}\left(\frac{\ell_{u_{n}}^{1-\alpha}}{\sqrt{a_{u_{n}} \ell_{u_{n}}}} \sup _{0 \leq i \leq k_{n}} \sup _{0 \leq T \leq 1}\left\|w\left(T l\left(u_{n+1}\right)+t_{i}+a_{u} \cdot\right)-w\left(t_{i}+a_{u} \cdot\right)\right\|_{\alpha} \geq \eta\right) \\
\leq & \sum_{i=0}^{k_{n}} C_{r, p}\left(\frac{\ell_{u_{n}}^{1-\alpha}}{\sqrt{a_{u_{n}} \ell_{u_{n}}}} \sup _{0 \leq T \leq 1}\left\|w\left(T l\left(u_{n+1}\right)+t_{i}+a_{u} \cdot\right)-w\left(t_{i}+a_{u} \cdot\right)\right\|_{\alpha} \geq \eta\right) \\
\leq & \sum_{i=0}^{k_{n}} \sum_{j=0}^{\left[\frac{a_{u}}{l\left(u_{n+1}\right)}\right]} C_{r, p}\left\{\frac{\ell_{u_{n}}^{1-\alpha}}{\sqrt{a_{u_{n} \ell_{u_{n}}}}} \frac{a_{u}^{\alpha}}{\left(l\left(u_{n+1}\right)\right)^{\alpha}} \mathcal{W}(j, n) \geq \eta\right\},
\end{aligned}
$$

where $\mathcal{W}(j, n)=\sup _{0 \leq T \leq 1} \| w\left(T l\left(u_{n+1}\right)+t_{i}+j l\left(u_{n+1}\right)+l\left(u_{n+1}\right) \cdot\right)-w\left(t_{i}+j l\left(u_{n+1}\right)+\right.$ $\left.l\left(u_{n+1}\right) \cdot\right) \|_{\alpha}$. 
We have

$$
\begin{aligned}
& C_{r, p}\left\{\ell_{u_{n}}^{1-\alpha} \sup _{0 \leq i \leq k_{n}} \sup _{0 \leq s \leq l\left(u_{n+1}\right)} \beta_{u_{n}}\left\|w\left(s+t_{i}+a_{u} \cdot\right)-w\left(t_{i}+a_{u} \cdot\right)\right\|_{\alpha} \geq \eta\right\} \\
\leq & \sum_{i=0}^{k_{n}} \sum_{j=0}^{\left[\frac{a_{u}}{l\left(u_{n}+1\right)}\right]} C_{r, p}\left\{\frac{\ell_{u_{n}}^{1-\alpha}}{\sqrt{a_{u_{n}} \ell_{u_{n}}}} \frac{a_{u}^{\alpha}}{\left(l\left(u_{n+1}\right)\right)^{\alpha}} \mathcal{W}(j, n) \geq \eta\right\} \\
\leq & \sum_{j=0}^{\left[\frac{a_{u}}{l\left(u_{n+1}\right)}\right]} \sum_{i=0}^{k_{n}} C_{r, p}\left\{\frac{\ell_{u_{n}}^{\frac{1}{2}-\alpha}}{\ell_{u_{n}}} \theta^{\alpha-\frac{1}{2}} \frac{\ell_{u_{n}}}{\ell_{u_{n+1}}} \frac{1}{\sqrt{l\left(u_{n+1}\right)}} \mathcal{W}(j, n) \geq \eta\right\} \\
\leq & \sum_{j=0}^{\left[\frac{a_{u}}{l\left(u_{n+1}\right)}\right]} \sum_{i=0}^{k_{n}} C_{r, p}\left\{\frac{1}{\ell_{u_{n}}^{\frac{1}{2}+\alpha}} \theta^{\alpha-\frac{1}{2}} \frac{1}{\sqrt{l\left(u_{n+1}\right)}} \mathcal{W}(j, n) \geq \eta\right\}
\end{aligned}
$$

and

$$
\begin{aligned}
& \mu\left\{\frac{\theta^{\alpha-\frac{1}{2}}}{\ell_{u_{n}}^{\frac{1}{2}+\alpha}} \frac{1}{\sqrt{l\left(u_{n+1}\right)}} \mathcal{W}(j, n) \geq \eta\right\} \\
= & \mu\left\{\frac{\sqrt{2} \theta^{\alpha-1 / 2}}{\ell_{u_{n}}^{\frac{1}{2}+\alpha}} \sup _{0 \leq T \leq 1}\left\|w\left(\frac{T}{2}+\frac{1}{2} \cdot\right)-w\left(\frac{1}{2} \cdot\right)\right\|_{\alpha} \geq \eta\right\}=\mu\left\{\frac{\sqrt{2} \theta^{\alpha-1 / 2}}{\ell_{u_{n}}^{\frac{1}{2}+\alpha}} w \in A\right\},
\end{aligned}
$$

where $A=\left\{f \in \mathcal{C}^{\alpha, 0}: \sup _{0 \leq t \leq 1}\left\|f\left(\frac{1}{2} t+\frac{1}{2} \cdot\right)-f\left(\frac{1}{2} \cdot\right)\right\|_{\alpha} \geq \eta\right\}$. Since $\inf _{f \in A} I(f) \geq \frac{\eta^{2}}{32}$, by Theorem 2.1 in $[4$, for $n$ large enough, we obtain

$$
\begin{aligned}
& C_{r, p}\left\{\frac{1}{\ell_{u_{n}}^{\frac{1}{2}+\alpha}} \theta^{\alpha-\frac{1}{2}} \frac{1}{\sqrt{l\left(u_{n+1}\right)}} \mathcal{W}(j, n)>\eta\right\} \\
& \leq \exp \left(-\frac{\eta^{2}}{128 \theta^{\alpha-1 / 2}} \ell_{u_{n}}^{2 \alpha} \ell_{u_{n}}\right)=\left(\frac{a_{u_{n}}}{u_{n} \log u_{n}^{-1}}\right)^{\frac{\eta^{2}}{128 \theta^{\alpha-1 / 2}} \ell_{u_{n}}^{2 \alpha}} .
\end{aligned}
$$

Taking into account that $\log \frac{u_{n} \log u_{n}^{-1}}{a_{u_{n}}} \rightarrow \infty$ as $n \rightarrow \infty$, we obtain

$$
\sum_{n} \frac{a_{u_{n}}}{l\left(u_{n+1}\right)} \frac{u_{n}+l\left(u_{n+1}\right)}{l\left(u_{n+1}\right)}\left(\frac{a_{u_{n}}}{u_{n} \log u_{n}^{-1}}\right)^{\frac{\eta^{2}}{128 \theta^{\alpha-1 / 2}} \ell_{u_{n}}^{2 \alpha}}<\infty .
$$

By Borel-Cantelli's Lemma,

$\limsup _{n \rightarrow \infty} \ell_{u_{n}}^{1-\alpha} \sup _{0 \leq i \leq k_{n}} \sup _{0 \leq s \leq l\left(u_{n+1}\right)} \beta_{u_{n}}\left\|w\left(s+t_{i}+a_{u} \cdot\right)-w\left(t_{i}+a_{u} \cdot\right)\right\|_{\alpha}=0, \quad C_{r, p}-q . s$.

By (3.3), (3.4) and (3.5), we obtain

(3.6) $\liminf _{n \rightarrow \infty} \ell_{u_{n}}^{1-\alpha} \inf _{t \in\left[0, u_{n}-a_{u_{n+1}}\right]}\left\|\beta_{u_{n}}\left(w\left(t+a_{u} \cdot\right)-w(t)\right)\right\|_{\alpha} \geq(k(\alpha))^{\gamma}, \quad C_{r, p}-$ q.s.

Let $\phi_{t, u}(s)=\beta_{u}\left(w\left(t+a_{u} s\right)-w(t)\right), s \in[0,1], t \in\left[0, u-a_{u}\right]$, for $u \in\left(u_{n+1}, u_{n}\right]$. Then

$$
\begin{aligned}
\inf _{t \in\left[0, u-a_{u}\right]}\left\|\phi_{t, u}(\cdot)\right\|_{\alpha} & \geq \inf _{t \in\left[0, u_{n}-a_{u_{n+1}}\right]} \frac{\beta_{u}}{\beta_{u_{n}}}\left\|\phi_{t, u_{n}}\left(\frac{a_{u}}{a_{u_{n}}} \cdot\right)\right\|_{\alpha} \\
& \geq \inf _{t \in\left[0, u_{n}-a_{u_{n+1}}\right]} \beta_{u_{n}}\left\|w\left(t+a_{u} \cdot\right)-w(t)\right\|_{\alpha} .
\end{aligned}
$$


Thus

$$
\ell_{u}^{1-\alpha} \inf _{t \in\left[0, u-a_{u}\right]}\left\|\phi_{t, u}(\cdot)\right\|_{\alpha} \geq \ell_{u_{n}}^{1-\alpha} \inf _{t \in\left[0, u_{n}-a_{u_{n+1}}\right]} \beta_{u_{n}}\left\|w\left(t+a_{u} \cdot\right)-w(t)\right\|_{\alpha} .
$$

We finally obtain

$$
\liminf _{u \rightarrow 0} \ell_{u}^{1-\alpha} \inf _{t \in\left[0, u-a_{u}\right]}\left\|\phi_{t, u}(\cdot)\right\|_{\alpha} \geq(k(\alpha))^{\gamma}, \quad C_{r, p}-q . s .
$$

Lemma 3.4. Letting $k(\alpha)$ be as in (2.1), we have $\liminf _{u \rightarrow 0} \ell_{u}^{1-\alpha} \inf _{t \in\left[0,1-\frac{a_{u}}{u}\right]}\left\|\beta_{u} \Delta(t, u)\right\|_{\alpha}$ $\leq(k(\alpha))^{\gamma}, \quad C_{r, p}-q . s$.

Proof. Step 1: Let $\lim _{u \rightarrow 0} \frac{\log \left(\frac{u}{a_{u}}\right)}{\log \log u^{-1}}<\infty$. Set $\rho=\lim _{u \rightarrow 0} \frac{a_{u}}{u}$.

Case 1. Letting $\rho<1$, we can define $u_{n}$ as follows: $u_{n+1}=u_{n}-a_{u_{n}}, u_{1}=\frac{1}{2}$. Then $\left\{\left\|\beta_{u_{n}} \Delta\left(1-\frac{a_{u_{n}}}{u_{n}}, u_{n}\right)-f\right\|_{\alpha}>\varepsilon\right\}(n \geq 1)$ are independent. We can prove that $u_{n} \rightarrow 0$ as $n \rightarrow \infty$. Taking $k=[r]+1$, by Lemma 2.3 , we have

$$
\begin{aligned}
& C_{r, p}\left(\bigcap_{n=m_{0}}^{l}\left(\ell_{u_{n}}^{1-\alpha}\left\|\beta_{u_{n}} \Delta\left(1-\frac{a_{u_{n}}}{u_{n}}, u_{n}\right)\right\|_{\alpha} \geq(k(\alpha))^{\gamma}(1+2 \varepsilon)\right)\right)^{1 / p} \\
& =C_{r, p}\left(\bigcap_{n=m_{0}}^{l}\left(\left\|\frac{1}{\sqrt{\ell_{u_{n}}}} \frac{\Delta\left(1-\frac{a_{u_{n}}}{u_{n}}, u_{n}\right)}{\sqrt{a_{u_{n}}}}\right\|_{\alpha} \geq \frac{(k(\alpha))^{\gamma}(1+2 \varepsilon)}{\ell_{u_{n}}^{1-\alpha}}\right)\right)^{1 / p} \\
& \leq c l^{k}\left(\frac{k(\alpha)^{\gamma} \varepsilon}{\ell_{u_{l}}^{1-\alpha}}\right)^{-2 k^{2}-k} \mu\left(\bigcap_{n=m_{0}}^{l}\left(\left\|\frac{\Delta\left(1-\frac{a_{u_{n}}}{u_{n}}, u_{n}\right)}{\sqrt{\ell_{u_{n}}} \sqrt{a_{u_{n}}}}\right\|_{\alpha} \geq \frac{k(\alpha)^{\gamma}(1+\varepsilon)}{\ell_{u_{n}}^{1-\alpha}}\right)\right)^{\frac{1}{q_{2}}} \\
& \leq c l^{k}\left(\frac{k(\alpha)^{\gamma} \varepsilon}{\ell_{u_{l}}^{1-\alpha}}\right)^{-2 k^{2}-k} \prod_{n=m_{0}}^{l}\left(1-\mu\left(\left\|\frac{\Delta\left(1-\frac{a_{u_{n}}}{u_{n}}, u_{n}\right)}{\sqrt{\ell_{u_{n}}} \sqrt{a_{u_{n}}}}\right\|_{\alpha}<\frac{k(\alpha)^{\gamma}(1+\varepsilon)}{\ell_{u_{n}}^{1-\alpha}}\right)\right)^{\frac{1}{q_{2}}} ;
\end{aligned}
$$

moreover, by a small deviation, for $n$ large enough, we have

$$
\mu\left(\left\|\frac{1}{\sqrt{\ell_{u_{n}}}} \frac{\Delta\left(1-\frac{a_{u_{n}}}{u_{n}}, u_{n}\right)}{\sqrt{a_{u_{n}}}}\right\|_{\alpha}<\frac{(k(\alpha))^{\gamma}(1+\varepsilon)}{\ell_{u_{n}}^{1-\alpha}}\right) \geq\left(\frac{a_{u_{n}}}{u_{n} \log u_{n}^{-1}}\right)^{\sigma_{1}},
$$

where $\sigma_{1}=\frac{1}{(1+\varepsilon)^{1 / \gamma}}+\sigma_{0}<1$, for some $\sigma_{0}>0$. Thus

$$
\begin{aligned}
& C_{r, p}\left(\bigcap_{n=m_{0}}^{l}\left(\ell_{u_{n}}^{1-\alpha}\left\|\beta_{u_{n}} \Delta\left(1-\frac{a_{u_{n}}}{u_{n}}, u_{n}\right)\right\|_{\alpha} \geq(k(\alpha))^{\gamma}(1+2 \varepsilon)\right)\right)^{1 / p} \\
\leq & c l^{k}\left(\frac{\ell_{u_{l}}^{1-\alpha}}{\varepsilon(k(\alpha))^{\gamma}}\right)^{2 k^{2}+k} \exp \left(-\frac{1}{q_{2}} \sum_{n=m_{0}}^{l} \ell_{u_{l}}^{-\sigma_{1}}\right) .
\end{aligned}
$$

Note that for $l$ large enough, there exists $C>0$ such that

$$
\sum_{n=m_{0}}^{l} \ell_{u_{l}}^{-\sigma_{1}}>C\left(\log u_{l}^{-1}\right)^{\varepsilon^{\prime}}
$$

where $\varepsilon^{\prime}=1-\sigma_{1}>0$. 
Since $\lim _{u \rightarrow 0} \frac{\log \left(u a_{u}^{-1}\right)}{\log \log u^{-1}}<\infty$, for some $0<M<\infty$, we have $u / a_{u} \leq\left(\log u^{-1}\right)^{M}$. Taking $\theta>2 / \varepsilon^{\prime}, u_{0}=e^{-\left(\log l_{0}\right)^{\theta}}$, for $l_{0}$ large enough, we can prove that

$$
\log u_{l}^{-1} \geq(\log l)^{\theta}, \text { as } l \geq l_{0} \text {; }
$$

thus we get

$$
\left(\log u_{l}^{-1}\right)^{\varepsilon^{\prime}}>(\log l)^{2} .
$$

We can also prove that when $l$ is large enough, there exists a constant $c_{1}>0$ such that $c_{1} l \geq \log u_{l}^{-1}$. Thus we have

$$
\begin{aligned}
& C_{r, p}\left(\bigcap_{n=m_{0}}^{l}\left(\ell_{u_{n}}^{1-\alpha}\left\|\beta_{u_{n}} \Delta\left(1-\frac{a_{u_{n}}}{u_{n}}, u_{n}\right)\right\|_{\alpha} \geq(k(\alpha))^{\gamma}(1+2 \varepsilon)\right)\right)^{1 / p} \\
\leq & c l^{k} L L\left(u_{l}^{-1}\right)^{(1-\alpha)\left(4 k^{2}+2 k\right)} \exp \left(-\frac{C}{q_{2}}\left(\log u_{l}^{-1}\right)^{\varepsilon^{\prime}}\right) \\
\leq & \frac{c_{2} l^{k}(\log l)^{(1-\alpha)\left(4 k^{2}+2 k\right)}}{\exp \left(\frac{C}{q_{2}}(\log l)^{2}\right)} \rightarrow 0, \quad l \rightarrow \infty
\end{aligned}
$$

where $c_{2}=c_{2}\left(k, p, q_{1}, k(\alpha), \alpha\right)$. We get

$$
C_{r, p}\left\{\bigcup_{l=1}^{\infty} \bigcap_{n=l}^{\infty}\left(\ell_{u_{n}}^{1-\alpha}\left\|\beta_{u_{n}} \Delta\left(1-\frac{a_{u_{n}}}{u_{n}}, u_{n}\right)\right\|_{\alpha} \geq(k(\alpha))^{\gamma}(1+2 \varepsilon)\right)\right\}=0 .
$$

Consequently,

$$
\liminf _{u \rightarrow 0} \ell_{u}^{1-\alpha}\left\|\beta_{u} \Delta\left(1-\frac{a_{u}}{u}, u\right)\right\|_{\alpha} \leq(k(\alpha))^{\gamma}, \quad C_{r, p}-q . s .
$$

Case 2. Let $\rho=1$; then $a_{u}=u$. See Theorem 2.1.

Step 2: Let $\lim _{u \rightarrow 0} \frac{\log \frac{u}{a_{u}}}{\log \log u^{-1}}=\infty$. The conclusion follows from Lemma 3.6.

We have proven (3.1). We are now in a position to prove (3.2).

Lemma 3.5. Let $k(\alpha)$ be as in (2.1). There exists a decreasing sequence $u_{n} \rightarrow$ $0(n \rightarrow \infty)$ such that

$$
\limsup _{n \rightarrow \infty} \ell_{u_{n}}^{1-\alpha} \inf _{t \in\left[0, u_{n+1}-a_{u_{n}}\right]}\left\|\beta_{u_{n}}\left(w\left(t+a_{u} s\right)-w(t)\right)\right\|_{\alpha} \leq(k(\alpha))^{\gamma}, \quad C_{r, p}-q . s .
$$

Proof. Since $\lim _{u \rightarrow 0} \frac{\log \frac{u}{a_{u}}}{\log \log u^{-1}}=\infty$, there exists a subsequence $\left\{u_{n} ; n \geq 1\right\}$ such that $\frac{u_{n}}{a_{u_{n}}}=n^{d^{\prime}}, d^{\prime}>1$. Letting $t_{i}=i a_{u_{n}}, i=0,1,2, \cdots, k_{n}=\left[\frac{u_{n+1}}{a_{u_{n}}}\right]-1$ and $h(n)=\frac{\log \frac{u_{n}}{a_{n}}}{\log \log u_{n}^{-1}}=\frac{\log n^{d^{\prime}}}{\log \log u_{n}^{-1}}$, we have $u_{n}^{-1}=\exp \left(n^{\frac{d^{\prime}}{h(n)}}\right)$ and $h(n) \rightarrow \infty$ as $n \rightarrow \infty$. Moreover, for any $\kappa>0$,

$$
\frac{n^{\kappa}}{\log u_{n}^{-1}} \rightarrow \infty, \quad n \rightarrow \infty
$$

and

$$
1 \leq \frac{u_{n}}{u_{n+1}}=\exp \left\{(n+1)^{\frac{d^{\prime}}{h(n+1)}}-n^{\frac{d^{\prime}}{h(n)}}\right\} \leq \exp \left\{n^{\frac{d^{\prime}}{h(n)}-1}\right\} \rightarrow 1, \quad n \rightarrow \infty
$$


Choose $\delta_{0}>0$ such that $\delta_{1}=\frac{1}{(1+\varepsilon)^{1 / \gamma}}+\delta_{0}<1$. Take $k=[r]+1$. By Lemma 2.3 and a small deviation, for $n$ large enough, we have

$$
\begin{aligned}
& C_{r, p}\left(\ell_{u_{n}}^{1-\alpha} \inf _{t \in\left[0, u_{n+1}-a_{u_{n}}\right]}\left\|\beta_{u_{n}}\left(w\left(t+a_{u} s\right)-w(t)\right)\right\|_{\alpha} \geq k(\alpha)^{\gamma}(1+2 \varepsilon)\right)^{1 / p} \\
\leq & C_{r, p}\left(\min _{0 \leq i \leq k_{n}}\left\|\beta_{u_{n}}\left(w\left(t_{i}+a_{u} s\right)-w\left(t_{i}\right)\right)\right\|_{\alpha} \geq \frac{k(\alpha)^{\gamma}(1+2 \varepsilon)}{\ell_{u_{n}}^{1-\alpha}}\right)^{1 / p} \\
\leq & \left(1+k_{n}\right)^{k}\left(\frac{\ell_{u_{n}}^{1-\alpha}}{k(\alpha)^{\gamma} \varepsilon}\right)^{2 k^{2}+k} \cdot \mu\left(\left\|\frac{w\left(t_{i}+a_{u} s\right)-w\left(t_{i}\right)}{\sqrt{a_{u_{n}}}}\right\|_{\alpha} \geq \frac{k(\alpha)^{\gamma}(1+\varepsilon)}{\ell_{u_{n}}^{\frac{1}{2}-\alpha}}\right)^{\frac{1+k_{n}}{q_{2}}} \\
\leq & \left.\left.\left(1+k_{n}\right)^{k}\left(\frac{\ell_{u_{n}}^{1-\alpha}}{k(\alpha)^{\gamma} \varepsilon}\right)^{2 k^{2}+k}\left(1-\mu\left(\|w(\cdot)\|_{\alpha}<\frac{k(\alpha)^{\gamma}(1+\varepsilon)}{\ell_{u_{n}}^{1 / 2-\alpha}}\right)\right)^{\frac{1+k_{n}}{q_{2}}}\right)^{\delta_{1}}\right)^{\frac{1+k_{n}}{q_{2}}}\left(1+k_{n}\right)^{k}\left(\frac{\ell_{u_{n}}^{1-\alpha}}{k(\alpha)^{\gamma} \varepsilon}\right)^{2 k^{2}+k}\left(1-\log u_{n}^{-1}\right)^{u_{n}} \\
\leq & c n^{k d^{\prime}}(\log n)^{\left(2 k^{2}+k\right)(1-\alpha)} \exp \left\{-\frac{1}{q_{2}}\left(\frac{a_{u_{n}}}{u_{n} \log u_{n}^{-1}}\right)^{\delta_{1}}\left[\frac{u_{n+1}}{a_{u_{n}}}\right]\right\}
\end{aligned}
$$

where $c>0$ is a constant. Choosing a suitable $d^{\prime}$, we have

$$
\sum_{n=1}^{\infty} c^{p} n^{p k d^{\prime}}(\log n)^{p\left(2 k^{2}+k\right)(1-\alpha)} \exp \left\{-\frac{1}{q_{2}}\left(\frac{a_{u_{n}}}{u_{n} \log u_{n}^{-1}}\right)^{\delta_{1}}\left[\frac{u_{n+1}}{a_{u_{n}}}\right]\right\}<\infty .
$$

By Borel-Cantelli's Lemma,

$$
\begin{aligned}
& \limsup _{n \rightarrow \infty}\left(\log \frac{u_{n} \log u_{n}^{-1}}{a_{u_{n}}}\right)^{1-\alpha} \inf _{t \in\left[0, u_{n+1}-a_{u_{n}}\right]}\left\|\beta_{u_{n}}\left(w\left(t+a_{u} s\right)-w(t)\right)\right\|_{\alpha} \\
\leq & k(\alpha)^{\gamma}(1+\varepsilon), \quad C_{r, p}-q . s .
\end{aligned}
$$

Lemma 3.6. Letting $k(\alpha)$ be as in (1), we have

$\limsup _{u \rightarrow 0}\left(\log \frac{u \log u^{-1}}{a_{u}}\right)^{1-\alpha} \inf _{t \in\left[0, u-a_{u}\right]}\left\|\beta_{u}\left(w\left(t+a_{u} s\right)-w(t)\right)\right\|_{\alpha} \leq k(\alpha)^{\gamma}, \quad C_{r, p}-q . s$.

Proof. Let $\psi_{t, u}(s)=\beta_{u}\left(w\left(t+a_{u} s\right)-w(t)\right), s \in[0,1], t \in\left[0, u-a_{u}\right]$. Let $u_{n}$ be defined as in Lemma 3.5. Then $\psi_{t, u}(s)=\frac{\beta_{u}}{\beta_{u_{n}}} \psi_{t, u_{n}}\left(\frac{a_{u}}{a_{u_{n}}} s\right)$. We have for $u \in$ $\left(u_{n+1}, u_{n}\right]$,

$$
\begin{aligned}
\inf _{t \in\left[0, u-a_{u}\right]}\left\|\beta_{u}\left(w\left(t+a_{u} s\right)-w(t)\right)\right\|_{\alpha} & =\inf _{t \in\left[0, u-a_{u}\right]}\left\|\frac{\beta_{u}}{\beta_{u_{n}}} \psi_{t, u_{n}}\left(\frac{a_{u}}{a_{u_{n}}} \cdot\right)\right\|_{\alpha} \\
& \leq \inf _{t \in\left[0, u_{n+1}-a_{u_{n}}\right]} \frac{\beta_{u}}{\beta_{u_{n}}}\left\|\psi_{t, u_{n}}\left(\frac{a_{u}}{a_{u_{n}}} .\right)\right\|_{\alpha} .
\end{aligned}
$$

Taking into account $\frac{\beta_{u_{n}}}{\beta_{u_{n+1}}} \rightarrow 1$ as $n \rightarrow \infty$, by Lemma 3.5, we obtain (3.8).

The conclusion (3.2) follows from Lemmas 3.3 and 3.6 . 


\section{REFERENCES}

[1] P. Baldi, G. Ben Arous and G. Kerkyacharian, Large deviations and the Strassen theorem in Hölder norm. Stochastic Processes and their Applications, 42 (1992), 171-180. MR:1172514 (93f:60034)

[2] P. Baldi and B. Roynette, Some exact equivalents for the Brownian motion in Hölder norm, Probab. Theory Relat. Fields, 93 (1992), 457-483. MR1183887 (94a:60117)

[3] M. Csörgö and P. Révesz, Strong approximations in probability and statistics, Academic Press, New York, 1981. MR666546 (84d:60050)

[4] Xiong Chen and N. Balakrishnan, Extensions of functional LIL w.r.t. $(r, p)$-capacities on Wiener space. Statistics \& Probab. Letters, 77 (2007), 468-473. MR 2339053

[5] Nina Gantert, An inversion of Strassen's law of the iterated logarithm for small time, Ann. Probab., 21 (1993), 1045-1049. MR1217579(94d:60130)

[6] Nobuo Yoshida, A large deviation principle for $(r, p)$-capacities on the Wiener space, Probab. Theory Relat. Fields, 94 (1993), 473-488. MR1201555 (94d:60041)

[7] Wensheng Wang, A generalization of functional law of the iterated logarithm for $(r, p)$ capacities on the Wiener space, Stochastic Processes and their Applications, 96 (2001), 1-16. MR:1856679 (2002f:60055)

School of Mathematics and Computing Science, Guilin University of Electronic Technology, Guilin 541004, People's Republic of China

E-mail address: liuyh1967cn@yahoo.com.cn

School of Mathematics and Computing Science, Guilin University of Electronic Technology, Guilin 541004, People's Republic of China 